\title{
BMJ Open Identification of vitamin C transporters in the human airways: a cross-sectional in vivo study
}

\author{
Nirina Larsson, ${ }^{1}$ Gregory D Rankin, ${ }^{1}$ Elif M Bicer, ${ }^{2}$ Ester Roos-Engstrand, ${ }^{1}$ \\ Jamshid Pourazar, ${ }^{1}$ Anders Blomberg, ${ }^{1}$ lan S Mudway, ${ }^{2}$ Annelie F Behndig ${ }^{1}$
}

To cite: Larsson N, Rankin GD, Bicer EM, et al. Identification of vitamin C transporters in the human airways: a cross-sectional in vivo study. BMJ Open 2015;5:e006979. doi:10.1136/bmjopen-2014006979

- Prepublication history and additional material is available. To view please visit the journal (http://dx.doi.org/ 10.1136/bmjopen-2014006979).

Received 23 October 2014 Revised 17 February 2015 Accepted 27 February 2015

\section{CrossMark}

\footnotetext{
${ }^{1}$ Department of Public Health and Clinical Medicine, Division of Medicine/ Respiratory Medicine and Allergy, Umeå University, Umeå, Sweden

${ }^{2}$ MRC-PHE Centre for Environment \& Health, Faculty of Life Sciences \& Medicine, Kings College London, London, UK
}

Correspondence to Dr lan S Mudway; ian.mudway@kcl.ac.uk

\section{ABSTRACT}

Objectives: Vitamin $\mathrm{C}$ is an important low-molecular weight antioxidant at the air-lung interface. Despite its critical role as a sacrificial antioxidant, little is known about its transport into the respiratory tract lining fluid (RTLF), or the underlying airway epithelial cells. While several vitamin $\mathrm{C}$ transporters have been identified, such as sodium-ascorbate cotransporters (SVCT1/2) and glucose transporters (GLUTs), the latter transporting dehydroascorbate, knowledge of their protein distribution within the human lung is limited, in the case of GLUTs or unknown for SVCTs.

Setting and participants: Protein expression of vitamin C transporters (SVCT1/2 and GLUT1-4) was examined by immunohistochemistry in endobronchial biopsies, and by FACS in airway leucocytes from lavage fluid, obtained from 32 volunteers; 16 healthy and 16 mild asthmatic subjects. In addition, antioxidant concentrations were determined in RTLF. The study was performed at one Swedish centre.

Primary and secondary outcome measures: The primary outcome measure was to establish the location of vitamin C transporters in the human airways.

As secondary outcome measures, RTLF vitamin C concentration was measured and related to transporter expression, as well as bronchial epithelial inflammatory and goblet cells numbers.

Results: Positive staining was identified for SVCT1 and 2 in the vascular endothelium. SVCT2 and GLUT2 were present in the apical bronchial epithelium, where SVCT2 staining was predominately localised to goblet cells and inversely related to RTLF vitamin C concentrations.

Conclusions: This experimental study is the first to demonstrate protein expression of GLUT2 and SVCT2 in the human bronchial epithelium. A negative correlation between SVCT2-positive goblet cells and bronchial RTLF vitamin C concentrations suggests a possible role for goblet cells in regulating the extracellular vitamin C pool.

\section{INTRODUCTION}

The antioxidant network within human respiratory tract lining fluids (RTLF) provides an important defence against inhaled oxidants ${ }^{1}$

\section{Strengths and limitations of this study}

- The present study is the first clinical investigation of vitamin $\mathrm{C}$ transporter expression in the airways of healthy subjects and patients with asthma.

- We present evidence of vitamin $C$ transporter (GLUT2 and SVCT2) protein expression in the bronchial epithelium, with evidence that SVCT2 expression is localised to goblet cells.

- Vitamin C is an important antioxidant in the lung, yet little is known about its transport into the respiratory tract lining fluid (RTLF). The presence of ascorbate and dehydroascorbate transporters (SVCT2 and GLUT2, respectively) within the pulmonary epithelium provides a potential salvage mechanism for vitamin $\mathrm{C}$, to prevent its oxidative loss from the surface of the lung. This helps to explain a hitherto significant gap in our knowledge of how vitamin $\mathrm{C}$ concentrations are maintained in vivo.

- Our observation of an inverse association between SVCT positive goblet cells and bronchial RTLF vitamin $\mathrm{C}$ suggests a possible role for goblet cells in the regulation of vitamin $C$ at the surface of the lung, however, the present study was not configured to pursue this further. Further research is required to confirm the role of goblet cells in airway vitamin $\mathrm{C}$ homeostasis.

- The current results only inform our understanding of vitamin $C$ in the proximal airways as we did not have tissue from the alveolar region.

and limits damage by inflammatory-derived oxidants during inflammation. ${ }^{1}{ }^{2}$ Ascorbate has been shown to be an important lowmolecular weight antioxidant within this compartment, with clear evidence that its concentration is reduced during and following acute inflammation, ${ }^{34}$ or as a direct consequence of exposure to inhaled oxidants. ${ }^{45}$ Consequently, numerous studies have attempted to augment RTLF antioxidant defences through high-dose vitamin C supplements. ${ }^{6-8}$ The rationale for this approach is based on the contention that ascorbate enters the RTLF from the plasma pool paracellularly ${ }^{9}$ and that by increasing 
plasma ascorbate, knock-on increases in RTLF concentrations will be achieved, conferring heightened protection against oxidative insults. However, the human supplementation studies performed to date have yielded modest or no protection against pulmonary oxidative stress. ${ }^{6-8}{ }^{10}$ In addition, in the majority of studies, while plasma concentrations of ascorbate have been increased, RTLF concentrations have either remained unchanged $^{7}{ }^{11}$ or have only demonstrated transient increases. ${ }^{12} 13$

These observations likely indicate the absence of a simplistic association between plasma and RTLF ascorbate pools. Either because ascorbate moving into the RTLF is rapidly lost by oxidation within this compartment, for which there is some support, ${ }^{12} 13$ or because it is sequestered into the cells of the airway, epithelial and resident inflammatory cells, effectively masking any underlying association between the plasma and RTLF pools. ${ }^{2}{ }^{14}$ Thus at this time, the fate of RTLF ascorbate is unknown. Continual loss of ascorbate at the air-lung interface would constitute a significant drain on the body's vitamin C reserves, with the oxidation of ascorbate to dehydroascorbate and its subsequent hydrolysis to 2,3-doketogulonic acid, resulting in the loss of its antioxidant function. ${ }^{15}$ Some form of recycling or salvage mechanism is therefore required to maintain pulmonary vitamin $\mathrm{C}$ reserves. As the RTLF does not appear to contain any functional dehydroascorbate reductase activity and as the concentration of glutathione ${ }^{16}$ within the compartment is insufficient to achieve this non-enzymatically, ${ }^{17}$ it appears that cellular uptake would be essential.

Two separate mechanisms exist for vitamin C uptake into cells: the high affinity, sodium-ascorbate cotransporters (SVCTs), ${ }^{18}$ (also known as SLC23A1-2 (solute carrier family)) and glucose transporters (GLUTs) ${ }^{19}$ (also known as SLC2A) the latter permitting the uptake of dehydroascorbate via facilitated diffusion. Dehydroascorbate transport, predominately by GLUT1 and $3,{ }^{19}$ and, to a lesser extent, by GLUT4 ${ }^{20}$ and GLUT2, ${ }^{21}$ is coupled to its rapid intracellular reduction back to ascorbate by a family of dehydroascorbate reductases, including glutaredoxin. ${ }^{14} 22$ The relevance of this salvage pathway in vivo is somewhat contentious due to the saturation of the transporters with glucose in most tissues. It is notable, however, that the concentrations of glucose in the RTLF are significantly lower than cellular and plasma concentrations: $0.4 \pm 0.2 \mathrm{mM}$, based on the analysis of breath condensate. ${ }^{23}$ To date, relatively few studies have examined the expression and cellular localisation of glucose transporters in the healthy lung, and these have returned contradictory results. Pezzulo examined GLUT transporter expression in primary human airway epithelial cells, demonstrating mRNA for GLUT1-12, but only protein expression for GLUT1 and 10 , in the basolateral and apical membranes, respectively. ${ }^{24}$ GLUT1 protein has also been detected in human lung homogenates by western blotting, ${ }^{25}$ in the absence of detectable GLUT3, 4 and 5. In the human lung adenocarcinoma epithelial cell line, H441, western blotting demonstrated GLUT2 and GLUT4 protein in non-polarised cells, with GLUT2 being present in the apical and basolateral membranes when the cells were polarised. ${ }^{26}$ This expression of GLUT2 was subsequently confirmed by immunohistochemistry in human airway biopsies, again with apical as well as basolateral expression. ${ }^{26}$

Messenger RNA for SVCT1 and SVCT2 has been reported in human bronchiolar epithelium, ${ }^{27}$ based on northern blotting, but there is currently no comparative information on protein expression and cellular localisation. $\mathrm{Jin}^{28}$ addressed this question in rats by immunohistochemistry, demonstrating SVCT1 and SVCT2 expression in the apical membrane of the tracheal, bronchial and alveolar epithelium. The resolution of the images did not allow a more detailed cellular attribution, however, and the relevance of these observations to non-ascorbate synthesisers, such as humans, remains unknown.

The primary aim of the present study was to establish the localisation of ascorbate (SVCT1/2) and dehydroascorbate (GLUT1-4) transporter proteins in the human bronchial epithelium and to determine whether vitamin C uptake by the respiratory epithelium played a role in the regulation of the extracellular ascorbate pool within the lung. We also evaluated the expression of SVCT $1 / 2$ and GLUT 1-3 transporters in airway leucocytes obtained from bronchoalveolar (BAL)-fluid, to provide a comprehensive examination of vitamin $\mathrm{C}$ transporter expression in the distal airways. The relationship between RTLF vitamin $\mathrm{C}$ concentrations, and transporter expression in the central and distal airways, was subsequently examined. Finally, as previous reports have indicated diminished RTLF ascorbate concentrations in asthmatics compared to the healthy, ${ }^{29} 30$ we also examined whether vitamin $\mathrm{C}$ handling at the air-lung interface differed between the two groups.

\section{METHODS}

Subjects

Healthy and asthmatic participants were invited to participate in the study through advertisement. Inclusion criteria were: age 18-40 years, no history of smoking, normal lung function (forced expiratory volume in $1 \mathrm{~s}$ $\left(\mathrm{FEV}_{1}\right)$ and forced vital capacity (FVC) of at least $80 \%$ of predicted, and a normal $\mathrm{FEV}_{1} / \mathrm{FVC}$ ratio) and absence of concomitant diseases, apart from allergy in the asthmatic group. All asthmatics had a positive history of allergy together with at least one positive skin prick test against a standard panel of common aeroallergens. In the asthmatic group, increased bronchial hyperresponsiveness to methacholine, with a provocative concentration causing a $20 \%$ fall in $\mathrm{FEV}_{1}<8 \mathrm{mg} / \mathrm{mL}$, was required. In total, 16 healthy subjects and 16 agematched patients with asthma were included in the study. Further details of the subject groups are summarised in the online supplementary material and within table 1 . Informed consent was obtained from all volunteers after verbal and written information. 


\section{Study design}

The study was performed outside the pollen season. Prior to the study visit, subjects fasted from midnight. All bronchoscopies were performed in the morning, and healthy and asthmatic participants were examined in a randomised order. Airway lavages and endobronchial biopsy sampling were performed as outlined previously. ${ }^{3}$ Further details are provided in the online supplementary material, with information on differential cell counts and lavage processing. Data on lung function and differential cell counts have been published previously. ${ }^{31}$

\section{Immunohistochemistry}

Endobronchial mucosal biopsies were processed and embedded into glycol methacrylate resin (Polyscience; Northampton, UK) ${ }^{3}$ - see online supplementary material. For the initial analyses, 2- $\mu \mathrm{m}$ sections were stained with monoclonal antibodies $(\mathrm{mAb})$ directed against neutrophil elastase (NE), tryptase (AA1) (DAKO, Glostrup, Denmark), eosinophil cationic protein (EG1), CD3 (lymphocytes) (Serotec, Oxford, UK) and glucose transporters 1-3 (R\&D Systems, Abingdon, UK) and 4 (Santa Cruz Biotechnology, Inc, Heidelberg, Germany). Rabbit polyclonal antibodies were used to stain the ascorbate transporters SVCT 1 and 2 (Santa Cruz Biotechnology, Inc, Heidelberg, Germany). Stained inflammatory cells were counted in the epithelium and in the submucosa, and expressed as cells $/ \mathrm{mm}$ and cells $/ \mathrm{mm}^{2}$, respectively. Epithelial staining for vitamin $\mathrm{C}$ transporters was quantified and expressed as per cent of stained epithelial area. Goblet cells were identified using a Periodic acid-Schiff (PAS) stain, ${ }^{32}$ expressed as cell number $/ \mathrm{mm}$ of epithelial length. To assess possible colocalisation of SVCT2 in PAS-positive cells, double immunostaining for PAS/ SVCT2 was performed. PAS/SVCT2-positive cells were expressed as the percentage of total goblet cells. Length and areas of the epithelium and submucosa were determined using the program LeicaQWin V3 (Leica Microsystems, Wetzlar, Germany). All analysis was performed in a blinded manner.

\section{Flow cytometry analysis}

Cells obtained from BAL-fluid were stained with antibodies against Peridinin Chlorophyll Protein (PerCP) conjugated antihuman CD3 (lymphocytes) (Becton Dickinson, San Jose, California, USA), phycoerytrin (PE) conjugated antihuman CD14 (monocytes/macrophages) (DAKO, Glostrup, Denmark) and fluorescein isothiocyanate (FITC) conjugated antihuman CD16b (neutrophils) (Serotec, Oxford, UK). Antibodies against SVCT1-2 and GLUT 1-3 were the same as those used for immunohistochemistry. Staining for flow cytometry analysis of SVCT1-2 and GLUT1-3 was performed in two steps using PE-conjugated rabbit antimouse secondary antibody (DAKO, Glostrup, Denmark) for GLUT 1-3 antibodies and FITC-conjugated swine antirabbit antibody (DAKO, Glostrup, Denmark) for SVCT1-2. Immunoreactivity was analysed using the BD FACS Calibur (Becton Dickinson, San Jose, California, USA) to evaluate the expression of transporters on different airway cell types. Further details are provided in the online supplementary material.

\section{Antioxidant analysis}

Vitamin C, ascorbate, dehydroascorbate, total glutathione (GSx), glutathione (GSH) and glutathione disulphide (GSSG) concentrations were determined in bronchial wash (BW) and BAL as previously described, ${ }^{3}{ }^{6}$ further details are provided in the online supplementary material. Cell-free lavage supernatants for vitamin C determinations were treated with the metal chelator desferoxamine mesylate (DES) and the synthetic antioxidant butylated hydroxytoluene (BHT), both at $2 \mathrm{mM} ; 5 \mu \mathrm{L}$ of each to $490 \mu \mathrm{L}$ of lavage, prior to storage at $-80^{\circ} \mathrm{C}$. Glutathione and urea determinations were performed in untreated lavage samples. Lavage and plasma urea concentrations were determined using the QuantiChrom urea assay kit (BioAssay Systems, Hayward). Urea dilution factors for BW and BAL for each subject were calculated as [urea $]_{\text {plasma/ }}$ [urea] $]_{\text {lavage }}$. Each subject's lavage measurements were multiplied by his/her unique dilution factors to correct for dilution, to derive bronchial and alveolar RTLF

Table 1 Demographics and clinical characteristics of the study population $n=32$

\begin{tabular}{lll}
\hline Characteristics & Healthy controls $\mathbf{n = 1 6}$ & Patients with asthma $\mathbf{n = 1 6}$ \\
\hline Male/female & $5 / 11$ & $10 / 6$ \\
Age (years) & $25(2)$ & $26(6)$ \\
BMl $\left(\mathrm{kg} / \mathrm{m}^{2}\right)$ & $23(2)$ & $24(3)$ \\
Methacholine $\mathrm{PC}_{20}(\mathrm{mg} / \mathrm{mL})$ & - & $1.1(0.7-4.4)$ \\
Skin prick test & Negative & Positive \\
Lung function (mean SD) & & $3.61(0.69)$ \\
$3 \mathrm{FEV}$ & $4.09(0.77)$ & $98(10)^{*}$ \\
FEV $\%$ pred & $105(8)$ & $4.48(1.22)$ \\
FVC & $4.81(1.05)$ & $101(13)$ \\
$\quad$ FVC \% pred & $106(9)$ & \\
\hline These data have been presented previously. & &
\end{tabular}


concentrations. Lavage samples for ascorbate determinations underwent deproteination with metaphosphoric acid (final concentration 5\%) and lipid extraction with heptane prior to analysis by reverse phase HPLC, with electrochemical detection $(500 \mathrm{mV}, 100 \mathrm{nA})$. An identical protocol was followed for the determination of vitamin $\mathrm{C}$ (ascorbate plus dehydroascorbate), with the exception that the samples underwent a reduction step with Tris (2-carboxylethyl) phosphine (TCEP) (Molecular Probes, Eugene, Oregon, USA). ${ }^{33}$ The dehydroascorbate concentration was determined by subtracting the measured ascorbate from the vitamin $\mathrm{C}$ concentration. Glutathione and glutathione disulfide concentrations were determined using the glutathione disulfide reductase-dithiobisnitrobenzoic acid recycling assay. ${ }^{36}$

\section{Statistics}

Antioxidant, inflammatory cell and immunohistochemistry data were not normally distributed, and are therefore described as medians with 25th and 75th centiles. Mann-Whitney U tests were employed to compare healthy and asthmatic data, with Spearman's rank correlation employed for analysis of association. Statistical analyses were performed using the IBM SPSS software, V.20 (SPSS, Armonk, New York, USA). A p value $<0.05$ was regarded as significant.

\section{RESULTS}

Bronchoscopies with endobronchial biopsy sampling, and collection of BW and BAL, were performed in all participants. Median BW and BAL recoveries were $42 \%$ and $72 \%$, with no significant differences between the two groups. In the asthmatic group, predicted $\mathrm{FEV}_{1}$ was significantly lower than in the healthy controls (table 1 ).
Consistent with their allergic status, patients with asthma had increased eosinophil and mast cell numbers in their BW and bronchial mucosa (see online supplementary table S1). Antioxidant concentrations in BW and BAL, corrected for sample dilution using the urea method, are presented in table 2, with no significant differences between the two groups. The ratio between dehydroascorbate and total vitamin $\mathrm{C}$ was significantly higher in the bronchial compared to distal airways; $89 \%$ (53-100) versus $13 \%$ (10-21) consistent with a more oxidative environment in proximal airways, with no significant differences between the two groups.

Sufficient biopsy material for immunohistochemistry analysis was obtained from all 32 participants. Endobronchial biopsies were stained for SVCT and GLUT transporters (table 3).

Staining with GLUT1 yielded a distinct cell membrane staining, most likely attributed to lymphocytes (figure 1). Positive membrane staining for GLUT2 was observed in bronchial epithelial cells, at their apical and their basolateral aspects, with little staining evident in the underlying basal cells (figure 1). Diffuse cytoplasmic staining was also seen, with a strong positive staining associated with the nuclear membrane. No differences in GLUT1 and 2 staining were evident between the healthy and patients with asthma (table 3). Staining for GLUT3 and 4 yielded inconclusive results due to unspecific background staining (data not shown). The FACS data examining GLUT1-3 demonstrated equivalent expression in BAL-fluid macrophages (CD14+) and neutrophils (CD16+), with evidence of a significant reduction of GLUT3 (1.4-fold) in lymphocytes (CD3+) obtained from asthmatic compared to healthy subjects, online supplementary table S2.

Positive staining was found in blood vessels for SVCT1 and 2, with localised staining for SVCT2 also observed in

Table 2 Low molecular weight antioxidants in bronchial wash (BW) and bronchoalveolar lavage (BAL) in patients with asthma and healthy individuals

\begin{tabular}{llcc}
\hline Antioxidant & $\begin{array}{l}\text { Healthy controls } \\
\mathbf{n = 1 6}\end{array}$ & $\begin{array}{l}\text { Patients with asthma } \\
\mathbf{n = 1 6}\end{array}$ & p Value \\
\hline Bronchial RTLF & & & \\
Ascorbate $(\mu \mathrm{M})$ & $10.5(0.0-71.0)$ & $6.39(0.0-97.9)$ & 0.92 \\
Dehydroascorbate $(\mu \mathrm{M})$ & $40.7(33.0-57.0$ & $38.5(21.2-53.0)$ & 0.58 \\
Vitamin C $(\mu \mathrm{M})$ & $52.3(37.3-138)$ & $49.1(23.0-149)$ & 0.49 \\
GSH $(\mu \mathrm{M})$ & $323(277-605)$ & $348(205-442)$ & 0.71 \\
GSSG $(\mu \mathrm{M})$ & $52.3(32.0-75.7)$ & $43.4(15.6-94.0)$ & 0.49 \\
GSx $(\mu \mathrm{M})$ & $508(350-757)$ & $465(287-622)$ & 0.10 \\
Alveolar RTLF & & & 0.65 \\
Ascorbate $(\mu \mathrm{M})$ & $103(81.2-150.1)$ & $79.4(55.7-119)$ & 0.14 \\
Dehydroascorbate $(\mu \mathrm{M})$ & $16.2(10.8-20.4)$ & $16.9(12.0-23.9)$ & 0.50 \\
Vitamin C $(\mu \mathrm{M})$ & $118(96.5-174)$ & $93.2(75.8-139)$ & 0.25 \\
GSH $(\mu \mathrm{M})$ & $124(101-181)$ & $126(46.6-219)$ & 0.84 \\
GSSG $(\mu \mathrm{M})$ & $21.3(17.2-37.5)$ & $19.3(12.1-26.5)$ & $177(81.4-244)$ \\
GSx $(\mu \mathrm{M})$ & $162(127-195)$ & & \\
\hline
\end{tabular}

Data are presented as median (IQR). Values are corrected for urea dilution.

GSH, reduced glutathione; GSSG, glutathione disulfide (oxidised glutathione); GSx, total glutathione; RTLF, respiratory tract lining fluid. 
Table 3 Quantification of SVCT 1 and 2 and GLUT 1 and 2 within the bronchial epithelium of healthy and mild patients with asthma $(n=32)$

\begin{tabular}{llll}
\hline $\begin{array}{l}\text { Vitamin } \mathbf{C} \text { transporters in } \\
\text { the bronchial epithelium }\end{array}$ & $\begin{array}{l}\text { Healthy controls } \\
\mathbf{n}=16\end{array}$ & $\begin{array}{l}\text { Patients with asthma } \\
\mathbf{n = 1 6}\end{array}$ & $\mathbf{p ~ V a l u e}$ \\
\hline SVCT 1 $(\%)$ & $0.19(0.11-0.82)$ & $0.10(0.04-0.26)$ & 0.30 \\
SVCT 2 $(\%)$ & $0.52(0.23-1.08)$ & $0.28(0.15-0.89)$ & 0.50 \\
GLUT 1 (\%) & $0.60(0.00-2.50)$ & $2.89(0.00-4.11)$ & 0.21 \\
GLUT 2 (\%) & $0.22(0.06-1.11)$ & $0.24(0.05-0.48)$ & 0.57 \\
\hline Staining is given as the percent positively stained area of the total epithelial area. Data are given as medians and IQR. & \\
GLUT, glucose transporters; SVCT, sodium-ascorbate cotransporters. & &
\end{tabular}

the apical epithelium (figure 2). No difference in the epithelial expression of SVCT1 or 2 was observed between the patients with asthma and healthy subjects (table 3). Similarly, no difference in SVCT staining was observed in leucocyte subsets by FACS (see online supplementary table S2). Within the bronchial epithelium, the localisation of SVCT2 staining to goblet-like cells was confirmed

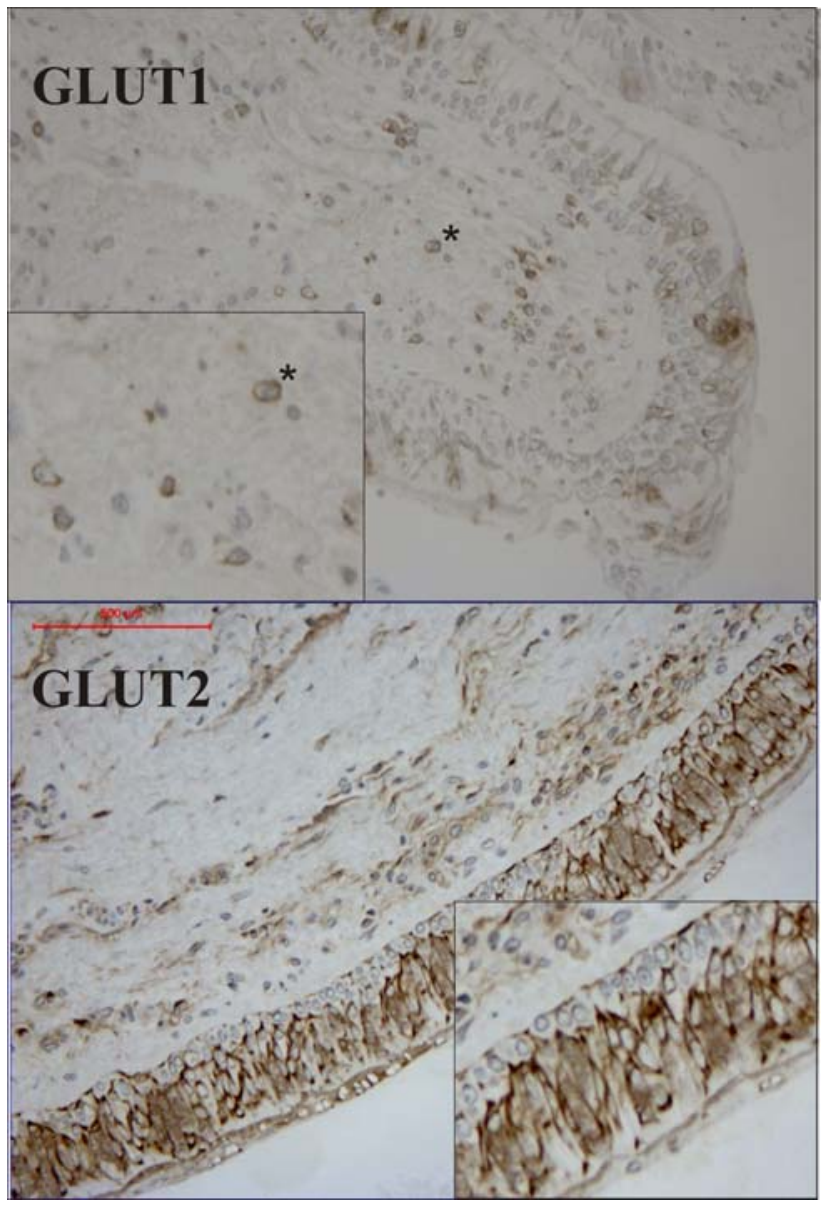

Figure 1 Immunohistochemical staining of glucose transporters (GLUT1) and (GLUT2) in bronchial biopsies obtained from a healthy subject. The upper panel shows GLUT1 staining with distinct cell membrane staining associated with lymphocytes within the submucosa $\left({ }^{*}\right)$. The lower panel demonstrates GLUT2 staining associated with the apical epithelium. The inset provides a magnification of the epithelium illustrating diffuse cytoplasmic staining, with more pronounced staining around the nuclear membrane. using PAS, to identify mucin-containing cells, and SVCT2 in consecutive and double-stained sections. These analyses were performed on 10 biopsies, five from the healthy group and five from the asthmatics (figure 2). The selected samples were chosen to encompass the full range of measured vitamin $\mathrm{C}$ concentrations within the bronchial RTLFs. No significant differences in the number of goblet cells or SVCT2-positive goblet cells were observed between the two groups (table 4).

There was a negative correlation between total vitamin $\mathrm{C}$ concentration in the bronchial RTLFs and goblet cells in bronchial biopsies: $\rho=-0.842, p<0.01$. This negative correlation remained when only SVCT2-positive goblet cells were considered: $\rho=-0.661, \mathrm{p}<0.05$ (figure 3). No other correlations were observed between the measured RTLF antioxidants with vitamin $\mathrm{C}$ transporters within the bronchial epithelium.

\section{DISCUSSION}

This study represents the first assessment of vitamin C transporter protein expression in human bronchial epithelium. In the proximal airways, SVCT2 expression was colocalised in mucin containing goblet cells, with the number of SVCT2-positive goblet cells inversely correlated with bronchial RTLF vitamin $\mathrm{C}$ concentrations. There was no difference in the expression SVCT1 and 2 within the bronchial mucosa between the healthy subjects and patients with asthma. With regard to GLUT expression, GLUT2 appeared the predominate glucose and, hence, potential dehydroascorbate transporter in the bronchial epithelium, with equivalent expression in the two groups. Overall, these data demonstrate the presence of ascorbate and dehydroascorbate transporters within the pulmonary epithelium, providing a salvage mechanism to prevent the loss of ascorbate from the surface of the lung.

In the present study, we did not observe evidence of lower ascorbate concentrations within the asthmatic RTLFs, in contrast to previous observations. ${ }^{3}{ }^{30}$ In the former study, ${ }^{3}$ a pronounced airway neutrophilia was present in patients with asthma compared to healthy subjects, which was absent here. In contrast, enhanced numbers of neutrophils were observed in the bronchial wash and bronchial submucosa of the healthy controls. However, in absolute terms, the neutrophilia was less 


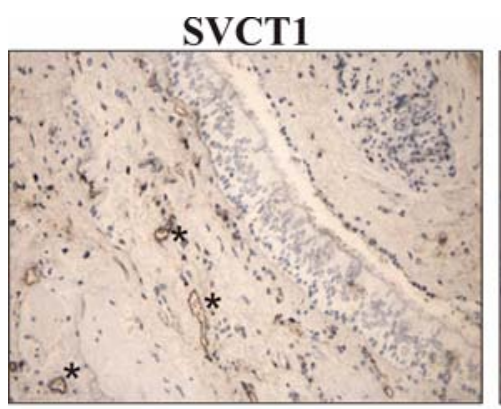

SVCT2

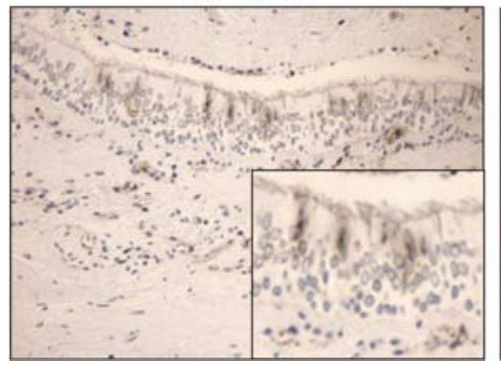

PAS

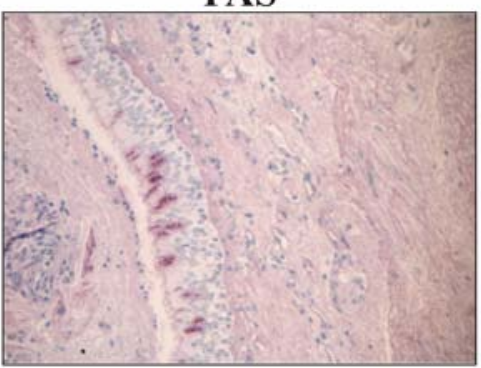

PAS

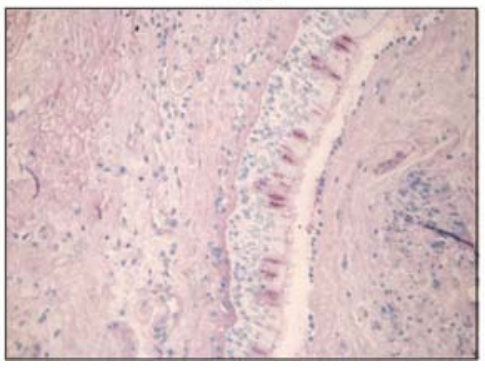

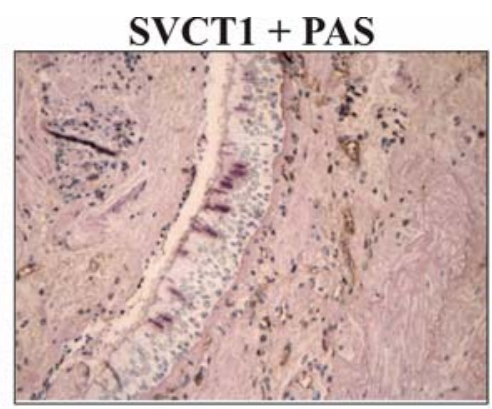

SVCT2 + PAS

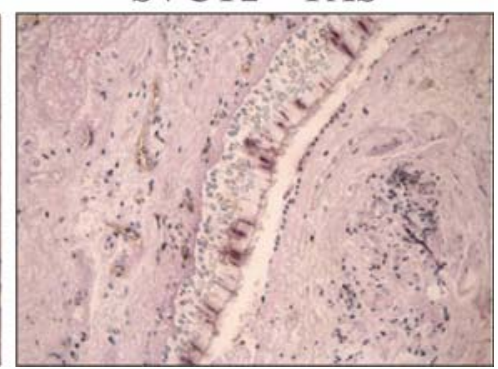

Figure 2 Immunohistochemical staining of sodium-ascorbate cotransporters (SVCT1) and (SVCT2) in bronchial biopsies. Upper left: SVCT1 was found present in blood vessels. Lower left: SVCT2 was found present in blood vessels and in the apical airway epithelium. Middle: Staining of mucins in goblet cells by Periodic acid-Schiff (PAS) staining. Upper and lower right: Consecutive sections from the same subject. SVCT2 apical epithelial staining largely localised to goblet cells.

marked than that previously observed in the asthmatic groups. ${ }^{3}$ Thus, there seems little relationship between the extent of neutrophilic or allergic inflammation and airway ascorbate concentrations, though the production of reactive oxygen species by activated phagocytes was not assessed in this or the previous studies.

It was also notable that a significant proportion of the RTLF vitamin C pool was present as its oxidised product, dehydroascorbate. In the alveolar samples the proportion of dehydroascorbate was 13.7 and $18.1 \%$ of the total vitamin $\mathrm{C}$ pool in the healthy subjects and patients with asthma, respectively; with a similar proportion of GSSG making up the GSx pool: 13.3 and 10.9\%. Though the concentrations of dehydroascorbate and GSSG were somewhat elevated, the measured concentrations were not implausible in a compartment lacking the intracellular mechanisms for regenerating ascorbate and glutathione. The proportion of dehydroascorbate to the vitamin C pool within the bronchial lavages was, however, extremely high in both groups: $77.8 \%$ (healthy) and $78.4 \%$ (asthmatics). This is a challenging observation, as the conditions likely to promote the formation of dehydroascorbate would also likely result in its further oxidation and, therefore, with current knowledge, we believe this result needs to be viewed with caution, especially as GSSG concentrations in the BW samples were not similarly increased. As the samples used for vitamin $\mathrm{C}$ analysis were pretreated with the metal chelator DES and synthetic antioxidant BHT to prevent erroneous sample oxidation during storage, these high concentrations are unlikely to be wholly attributable to erroneous oxidation during storage or sample processing. One explanation for the elevated concentrations of dehydroascorbate in human RTLFs is that ascorbate moving onto the surface of the lung is subject to oxidation, partly explaining the absence of a clear association between plasma and RTLF pools previously reported, ${ }^{33}$ the higher concentrations in the bronchial RTLFs reflecting a more oxidative environment. Previous supplementation studies have shown a rapid flux of ascorbate into the airways, ${ }^{12}{ }^{13}$ which is subsequently lost over a $24 \mathrm{~h}$ period. ${ }^{13}$ Schock et $a l^{12}$ demonstrated that ascorbate in induced sputum is rapidly oxidised in an azide-inhibitable manner, suggesting that endogenous peroxidases contribute to the oxidative losses observed. The RTLF has also been shown to contain appreciable concentrations of non-transferrinbound iron, ${ }^{34}$ consistent with a pro-oxidant environment. The problem with this contention is that if such losses were occurring across the large surface area of the lung, this would constitute a major drain on bodily vitamin $\mathrm{C}$ reserves. In the absence of a putative dehydroascorbate reductase within the RTLF, it would therefore be necessary to have mechanisms to rapidly transport ascorbate and dehydroascorbate into the pulmonary epithelium, to maintain antioxidant defences at the air-lung interface.

To detect and localise vitamin $\mathrm{C}$ transporters in the bronchial mucosa and in airway leucocytes obtained from BAL-fluid, commercially available antibodies were used. There is a lack of in vivo human studies on these transporters especially in regard to the lung. Most studies looking at tissue localisation have been conducted in animals that can synthesise vitamin C themselves. Much of the data accumulated on expression in particular cells have also been conducted using in vitro experiments, however, expression of the SVCTs has been shown to change depending on culture conditions and 
Table 4 Quantification of goblet cells and SVCT2-positive goblet cells within the bronchial epithelium of selected healthy and mild patients with asthma

\begin{tabular}{llcl}
\hline & $\begin{array}{l}\text { Goblet cells } \\
(\text { cells/mm) }\end{array}$ & $\begin{array}{l}\text { SVCT2-positive goblet } \\
\text { cells (cells/mm) }\end{array}$ & $\begin{array}{l}\text { Per cent SVCT2-positive } \\
\text { goblet cells }\end{array}$ \\
\hline Healthy subjects $(n=5)$ & $25.5(16.6-41.8)$ & $16.3(9.2-33.6)$ & $60.5(54.0-77.6)$ \\
Patients with asthma $(n=5)$ & $20.6(8.0-31.7)$ & $11.2(3.8-17.5)$ & $54.1(42.1-62.7)$ \\
p Value & 0.42 & 0.42 & 0.22 \\
Data are given as number of cells per mm epithelium and \% SVCT2-positive goblet cells. Data are presented as median (IQR). \\
SVCT, sodium-ascorbate cotransporters.
\end{tabular}

therefore is a factor to consider. In one paper addressing the distribution of SVCT1 and 2 transporters in rat liver, they found colocalisation of SVCT1 and 2 with endothelial cells. Of note, in that paper they used the same antibodies as in the present study. ${ }^{35}$

Dehydroascorbate uptake into cells occurs by facilitated diffusion through glucose transporters,
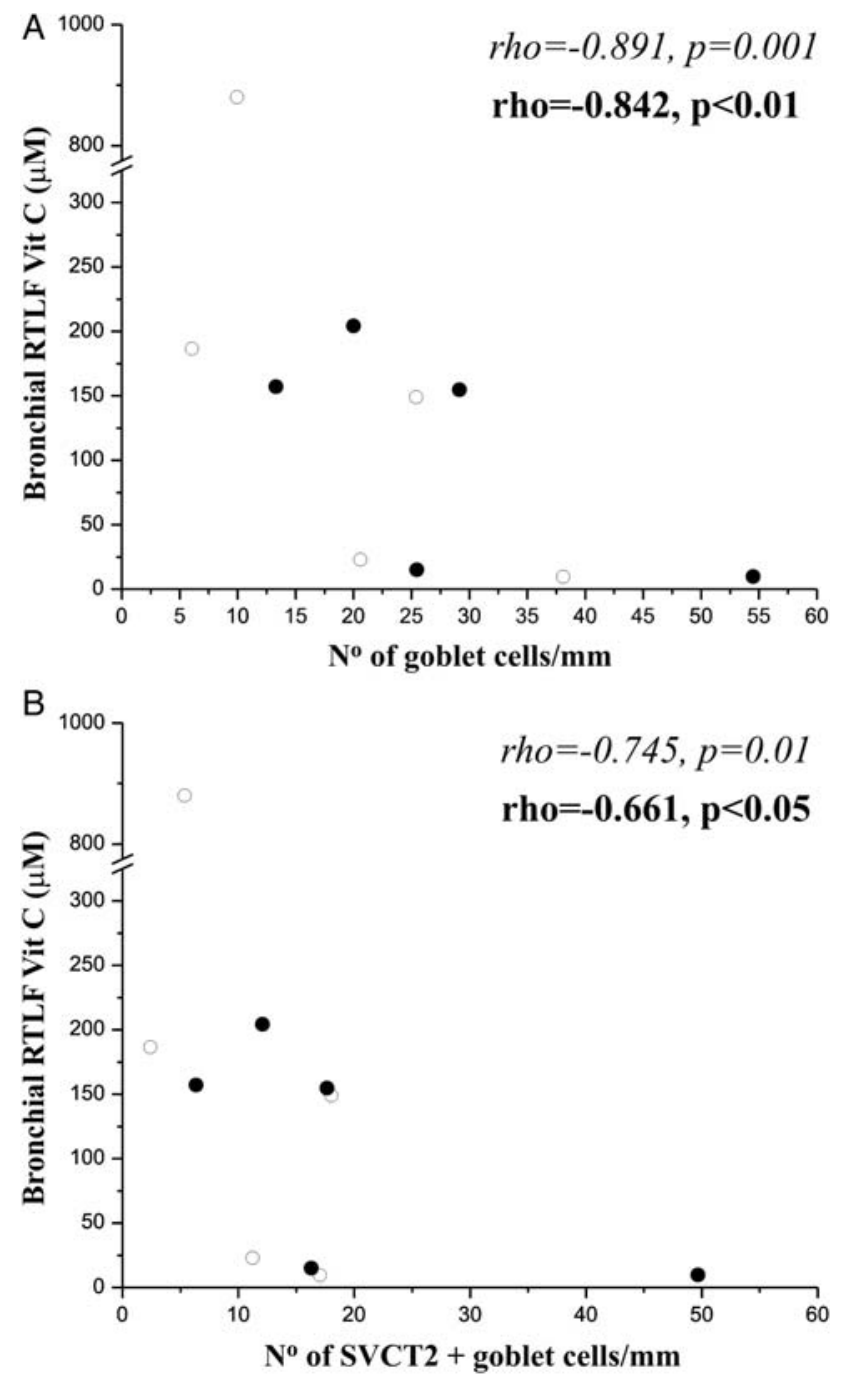

Figure 3 (A) Goblet cells in bronchial biopsies and total vitamin $C$ concentrations in bronchial wash show a negative correlation $(\rho=-0.842, p<0.01)$. (B) sodium-ascorbate cotransporters (SVCT2)+goblet cells in bronchial biopsies and total vitamin $\mathrm{C}$ concentrations in bronchial wash show a negative correlation $(r=-0.661, p<0.05)$. predominately GLUT1 and GLUT3, ${ }^{19}$ but with evidence that GLUT2 ${ }^{21}$ and $4^{20}$ can also mediate uptake. Knowledge concerning the epithelial expression of GLUTs in the lung, excluding lung carcinomas, is scarce, with conflicting evidence obtained from tissue biopsies and primary and immortalised human epithelial cells. ${ }^{23-26}$ Once internalised, dehydroascorbate is rapidly reduced to ascorbate by NADPH-dependent dehydroascorbate reductases. ${ }^{2}{ }^{14}$ It has been argued that dehydroascorbate uptake via facilitated glucose transporters is unlikely to play a major role under physiological conditions, as high cellular and extracellular glucose concentrations will block dehydroascorbate influx. Hence dehydroascorbate uptake has been argued only to occur during episodes of oxidative stress, where high local concentrations of dehydroascorbate exist near cells producing reactive oxygen species (ROS). This has been demonstrated in neutrophils undergoing oxidative burst, ${ }^{14}$ and in cells near the site of ROS production. ${ }^{2}$ While dehydroascorbate uptake by cells is typically antagonised by glucose, the RTLF has a low glucose concentration compared with other body compartments. ${ }^{23}$ This raises the possibility that dehydroascorbate formed in this compartment may be taken up by airway cells. In the current study we found cell immunoreactivity for GLUT2 expressed at the apical and also basolateral aspects of the columnar epithelial cells. A recent paper has demonstrated that GLUT2 plays an important role in dehydroascorbate uptake from the gut lumen, ${ }^{21}$ and it is possible that it performs a similar function in the lung.

SVCT1 and 2 have been shown to act as intracellular transporters of ascorbate, ${ }^{18}{ }^{27}$ SVCT1 being associated predominately with epithelial cells, ${ }^{27}$ and SVCT2 with a wider range of metabolically active cells. ${ }^{27}$ Although previous studies have demonstrated mRNA for both SVCT1 and 2 within human bronchiolar epithelium, ${ }^{27}$ no information is available on cellular distribution or protein expression. An examination of SVCT1 and 2 expression in the rat has shown immunoreactivity at the apical epithelium of the trachea, bronchi and alveoli. ${ }^{28}$ In vitamin C synthesisers, SVCT-2 has been shown to be essential, with knock-out mice dying, shortly after birth, of respiratory failure. ${ }^{36}$ Over expression of SVCT2 has also been shown to confer protection against paraquat-induced oxidative stress in transgenic mice expressing additional copies of the gene, associated with elevated pulmonary 
tissue ascorbate concentrations. ${ }^{37}$ These observations highlight the importance of vitamin $\mathrm{C}$ salvage mechanisms in the lung, which are likely even more significant in non-synthesising species such as man. Here we report for the first time evidence of pronounced SVCT2 protein expression within goblet cells of the bronchial epithelium. In addition, vitamin $\mathrm{C}$ concentrations were inversely correlated with the number of SVCT2-positive goblet cells, indicating a possible role for these cells in vitamin $\mathrm{C}$ homeostasis. These data are consistent with the ascorbate pool within the proximal airway being largely determined by its uptake into goblet cells. However, whether ascorbate can be released onto the airway surface from these cells under conditions of oxidative stress remains an area for future research. While vitamin $\mathrm{C}$ uptake mechanisms are well defined, less is known concerning potential efflux from cells. ${ }^{38}$ Transport through volume sensitive anion channels has been shown, ${ }^{39}$ as has the release of ascorbate-containing vesicles, ${ }^{40}$ and ascorbate-ascorbate homoexchange; ${ }^{41}$ though, to date, the information on these transport mechanisms remains limited. In light of these results, there is now a motivation to address this issue in goblet cells, especially as exposure to airway irritants such as diesel exhaust ${ }^{42}$ and ozone ${ }^{6}$ has been shown to elicit acute increases in ascorbate and vitamin $\mathrm{C}$ concentrations in upper airway secretions of human subjects. To date, these increases have tended to be related to alterations in the permeability of the bloodair barrier, but this has not been conclusively established. As goblet cells are often increased in asthmatic airways, one might therefore expect differences in cellular uptake to impact on RTLF ascorbate concentrations compared with the healthy population, that is, lower extracellular vitamin $\mathrm{C}$ concentrations due to increased uptake by goblet cells. This was not shown in the present study, with equivalent number of goblet cells in the two groups, possibly reflecting the relatively mild clinical status of the patients with asthma.

In the present study immunohistochemical characterisation of vitamin $\mathrm{C}$ transport expression was limited to the bronchial airways, and therefore the question of the regulation of the extracellular ascorbate pool in the alveolar region remains unresolved. We did attempt to gain some insight into vitamin $\mathrm{C}$ transport in the distal lung using FACS-based analysis of SVCT and GLUT transporters in the resident leucocyte population, recovered by bronchoalveolar lavage. Focusing on macrophages, neutrophils and lymphocytes, we observed no differences in SVCT1 and 2 expressions between the healthy subjects and patients with asthma. There was evidence of reduced GLUT3 expression in CD3+lymphocytes in asthmatics, but overall there was no clear relationship between the measured vitamin $\mathrm{C}$ concentrations and the SVCT and GLUT expression observed in BAL cells.

In conclusion, we have shown that the human bronchial epithelium has two potential salvage mechanisms, SVCT2 and GLUT2, allowing cellular uptake of ascorbate moving into the RTLF from the plasma pools, hence preventing its irreversible oxidation to 2,3-doketogulonic acid and loss of its antioxidant function. Further studies are warranted to explore vitamin $\mathrm{C}$ transporters in the alveolar region of the lung.

Acknowledgements The authors wish to thank Ann-Britt Lundström, Sofie Wennberg, Annika Johansson and Frida Holmström for their expert technical assistance, and the volunteers participating in this study.

Contributors NL was involved in study design, recruitment of participants, bronchoscopies, analysis of data and preparation of manuscript. GDR performed immunohistochemistry and was involved in preparation of manuscript. EMB carried out antioxidant analysis. ER-E took part in sample preparation FACS and immunohistochemistry. JP took part in sample preparation, analysis of inflammatory cell counts and immunohistochemistry. $A B$ was involved in study design, recruitment of participants, bronchoscopies, analysis of data and preparation of manuscript. ISM was involved in study design, statistics, analysis of data and preparation of manuscript and is the corresponding author. AFB took part in study design, recruitment of participants, bronchoscopies, analysis of data and preparation of manuscript.

Funding This study was funded by Swedish Heart-Lung Foundation; Umea University, Sweden; Västerbotten County Council, Sweden; VISARE NORR, Norrlandstingens regionförbund (Northern County Councils), Sweden.

\section{Competing interests None.}

\section{Patient consent Obtained.}

Ethics approval The study was approved by the local ethical review board at Umeå University and performed according to the declaration of Helsinki.

Provenance and peer review Not commissioned; externally peer reviewed.

Data sharing statement No additional data are available.

Open Access This is an Open Access article distributed in accordance with the Creative Commons Attribution Non Commercial (CC BY-NC 4.0) license, which permits others to distribute, remix, adapt, build upon this work noncommercially, and license their derivative works on different terms, provided the original work is properly cited and the use is non-commercial. See: http:// creativecommons.org/licenses/by-nc/4.0/

\section{REFERENCES}

1. Mudway IS, Kelly FJ. Ozone and the lung: a sensitive issue. Mol Aspects Med 2000;21:1-48.

2. Nualart FJ, Rivas $\mathrm{Cl}$, Montecinos VP, et al. Recycling of vitamin C by a bystander effect. J Biol Chem 2003;278:10128-33.

3. Mudway IS, Stenfors N, Blomberg A, et al. Differences in basal airway antioxidant concentrations are not predictive of individual responsiveness to ozone: a comparison of healthy and mild asthmatic subjects. Free Radic Biol Med 2001;31:962-74.

4. Mudway IS, Krishna MT, Frew AJ, et al. Compromised concentrations of ascorbate in fluid lining the respiratory tract in human subjects after exposure to ozone. Occup Environ Med 1999;56:473-81.

5. Kelly FJ, Blomberg A, Frew A, et al. Antioxidant kinetics in lung lavage fluid following exposure of humans to nitrogen dioxide. $\mathrm{Am} J$ Respir Crit Care Med 1996;154:1700-5.

6. Behndig AF, Blomberg A, Helleday R, et al. Antioxidant responses to acute ozone challenge in the healthy human airway. Inhal Toxicol 2009;21:933-42.

7. Mudway IS, Behndig AF, Helleday R, et al. Vitamin supplementation does not protect against symptoms in ozone-responsive subjects. Free Radic Biol Med 2006;40:1702-12.

8. Samet JM, Hatch GE, Horstman D, et al. Effect of antioxidant supplementation on ozone-induced lung injury in human subjects. Am J Respir Crit Care Med 2001;164:819-25.

9. May JM, Qu ZC, Qiao H. Transfer of ascorbic acid across the vascular endothelium: mechanism and self-regulation. Am J Physiol Cell Physiol 2009;297:C169-78.

10. Gomes EC, Allgrove JE, Florida-James G, et al. Effect of vitamin supplementation on lung injury and running performance in a hot humid, and ozone-polluted environment. Scand J Med Sci Sports 2011;21:e452-60. 
11. Hernandez M, Zhou H, Zhou B, et al. Combination treatment with high-dose vitamin $\mathrm{C}$ and alpha-tocopherol does not enhance respiratory-tract lining fluid vitamin $\mathrm{C}$ levels in asthmatics. Inhal Toxicol 2009;21:173-81.

12. Schock BC, Koostra J, Kwack S, et al. Ascorbic acid in nasal and tracheobronchial airway lining fluids. Free Radic Biol Med 2004:37:1393-401.

13. Behndig AF, Blomberg A, Helleday R, et al. Augmentation of respiratory tract lining fluid ascorbate concentrations through supplementation with vitamin C. Inhal Toxicol 2009;21:250-8.

14. Wang $\mathrm{Y}$, Russo TA, Kwon $\mathrm{O}$, et al. Ascorbate recycling in human neutrophils: induction by bacteria. Proc Natl Acad Sci USA 1997;94:13816-19.

15. Koshiishi I, Mamura Y, Liu J, et al. Degradation of dehydroascorbate to 2,3-diketogulonate in blood circulation. Biochim Biophys Acta 1998;1425:209-14.

16. van der Vliet A, O'Neill CA, Cross CE, et al. Determination of low-molecular-mass antioxidant concentrations in human respiratory tract lining fluids. Am J Physiol 1999;276:L289-96.

17. Winkler BS, Orselli SM, Rex TS. The redox couple between glutathione and ascorbic acid: a chemical and physiological perspective. Free Radic Biol Med 1994;17:333-49.

18. Bürzle M, Suzuki Y, Ackermann D, et al. The sodium-dependent ascorbic acid transporter family SLC23. Mol Aspects Med 2013;34:436-54.

19. Rumsey SC, Kwon O, Xu GW, et al. Glucose transporter isoforms GLUT1 and GLUT3 transport dehydroascorbic acid. J Biol Chem 1997;272:18982-9.

20. Rumsey SC, Daruwala R, Al-Hasani H, et al. Dehydroascorbic acid transport by GLUT4 in Xenopus oocytes and isolated rat adipocytes. $J$ Biol Chem 2000;275:28246-53.

21. Corpe CP, Eck P, Wang J, et al. Intestinal dehydroascorbic acid (DHA) transport mediated by the facilitative sugar transporters, GLUT2 and GLUT8. J Biol Chem 2013;288:9092-101.

22. Park JB, Levine M. Purification, cloning and expression of dehydroascorbic acid-reducing activity from human neutrophils: identification as glutaredoxin. Biochem J 1996;315:931-8.

23. Garnett JP, Baker EH, Baines DL. Sweet talk: insights into the nature and importance of glucose transport in lung epithelium. Eur Respir J 2012;40:1269-76.

24. Pezzulo AA, Gutiérrez J, Duschner KS, et al. Glucose depletion in the airway surface liquid is essential for sterility of the airways. PLOS ONE 2011;6:e16166.

25. Devaskar SU, de Mello DE. Cell-specific localization of glucose transporter proteins in mammalian lung. J Clin Endocrinol Metab 1996;81:4373-8.

26. Kalsi KK, Baker EH, Medina RA, et al. Apical and basolateral localisation of GLUT2 transporters in human lung epithelial cells. Pflugers Arch 2008;456:991-1003.
27. Tsukaguchi $\mathrm{H}$, Tokui $\mathrm{T}$, Mackenzie $\mathrm{B}$, et al. A family of mammalian Na+-dependent L-ascorbic acid transporters. Nature 1999;399: 70-5.

28. Jin SN, Mun GH, Lee JH, et al. Immunohistochemical study on the distribution of sodium-dependent vitamin $\mathrm{C}$ transporters in the respiratory system of adult rat. Microsc Res Tech 2005;68:360-7.

29. Kelly FJ, Mudway I, Blomberg A, et al. Altered lung antioxidant status in patients with mild asthma. Lancet 1999;354:482-3.

30. Kongerud J, Crissman K, Hatch G, et al. Ascorbic acid is decreased in induced sputum of mild asthmatics. Inhal Toxicol 2003;15:101-9.

31. Larsson N, Lundström SL, Pinto R, et al. Lipid mediator profiles differ between lung compartments in asthmatic and healthy humans. Eur Respir J 2014;43:453-63.

32. Cerri PS, Sasso-Cerri E. Staining methods applied to glyco methacrylate embedded tissue sections. Micron 2003;34:365-72.

33. Blomberg A, Mudway IS, Nordenhäll C, et al. Ozone-induced lung function decrements do not correlate with early airway inflammatory or antioxidant responses. Eur Respir J 1999;13:1418-28.

34. Gutteridge JM, Mumby S, Quinlan GJ, et al. Pro-oxidant iron is present in human pulmonary epithelial lining fluid: implications for oxidative stress in the lung. Biochem Biophys Res Commun 1996;220:1024-7.

35. Macias RI, Hierro C, de Juan SS, et al. Hepatic expression of sodium-dependent vitamin $C$ transporters: ontogeny, subtissular distribution and effect of chronic liver diseases. Br J Nutr 2011;106:1814-25.

36. Sotiriou S, Gispert S, Cheng J, et al. Ascorbic-acid transporter Slc23a1 is essential for vitamin $C$ transport into the brain and for perinatal survival. Nat Med 2002;8:514-17.

37. Harrison FE, Best JL, Meredith ME, et al. Increased expression of SVCT2 in a new mouse model raises ascorbic acid in tissues and protects against paraquat-induced oxidative damage in lung. PLOS ONE 2012;7:e35623.

38. Lane DJ, Lawen A. Ascorbate and plasma membrane electron transport-enzymes vs efflux. Free Radic Biol Med 2009;47:485-95.

39. Davis KA, Samson SE, Best K, et al. Ca2+-mediated ascorbate release from coronary artery endothelial cells. Br J Pharmacol 2006;147:131-9.

40. von Zastrow M, Tritton TR, Castle JD. Identification of L-ascorbic acid in secretion granules of the rat parotid gland. $J$ Biol Chem 1984:259:11746-50.

41. May JM, Qu ZC. Ascorbic acid efflux and re-uptake in endothelial cells: maintenance of intracellular ascorbate. Mol Cell Biochem 2009;325:79-88.

42. Blomberg A, Sainsbury C, Rudell B, et al. Nasal cavity lining fluid ascorbic acid concentration increases in healthy human volunteers following short term exposure to diesel exhaust. Free Radic Res 1998;28:59-67. 